are at the same risk for occult bacteremia as patients with fever alone, and should receive the same attention with regard to blood cultures. My colleague, Dr. Subhash Chaudhary, Head of the Division of Pediatric Infectious Disease at SIU School of Medicine, commented on the indications for blood culture in young children with fever but with no recognized focus of infection: "a child who looks sick and/or has a WBC of 20,000 or more should receive a blood culture and initial treatment with an antibiotic effective against pneumococcus and $\mathrm{H}$ influenza type $b$ pending the isolation of an organism."

\title{
NONTRAUMATIC LUMBAR PUNCTURE
}

A technique and formula for avoiding the traumatic spinal tap is reported from the Medical College of Wisconsin, Milwaukee, WI. To increase the accuracy and to minimize the frequency of traumatic puncture, the authors conducted a 12-month prospective analysis of 158 children of various ages in whom this diagnostic procedure was performed during the evaluation of an acute illness. The right lateral decubitus position was used and the needle was inserted perpendicularly in the L-3 to L-4 vertebral interspace. After CSF was collected, the needle was marked at the skin line and the length inserted was measured. The patient's age, weight, and height were used to calculate the body-surface area in square meters. The body-surface area showed the highest correlation with the depth of lumbar puncture. Linear regression for surface area provided a simple formula to estimate the depth of puncture to within 5 $\mathrm{mm}$ in young children of all ages:

$$
\text { Depth }=0.77 \mathrm{~cm}+2.56\left(\mathrm{~m}^{2}\right)
$$

(Bonadio WA et al. Estimating lumbar-puncture depth in

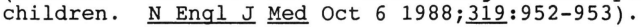

COMMENT. Insertion of the needle too deeply with puncture of venous plexuses in the anterior wall of the vertebral wall is the most common error. The avoidance of traumatic lumbar puncture by the use of this simple calculation and formula should facilitate the diagnosis of meningitis and reduce the risk of iatrogenic meningitis resulting from blood contamination of a previously sterile CSF in the patient with bacteremia. The authors are to be congratulated on their attempt to introduce some mathematical accuracy into a commonly "hit or miss" procedure.

\section{INVOLUNTARY MOVEMENTS}

\section{SYDENHAM'S CHOREA THERAPY}

Five patients with chorea successfully treated with carbamazepine at plasma levels of $6.5-8.8 \mathrm{mcg} / \mathrm{ml}$ are reported from the Dept of Pediatrics, Child Neurology and EEG Service, Hospital Infantil Vall d'Hebron, Autonomous University, 